\title{
Amyloid PET imaging: a challenge for research in clinical neuroimaging
}

\author{
Kirk Frey $\cdot$ Daniela Perani
}

Received: 22 January 2015/Accepted: 24 January 2015/Published online: 13 February 2015

(C) Italian Association of Nuclear Medicine and Molecular Imaging 2015

The development of amyloid targeting PET radioligands is one of the most challenging research and clinical neuroimaging endeavours. The first radiopharmaceutical developed by investigators at the University of Pittsburgh, a derivative of a histopathologic dye (thioflavin-S) used to identify fibrillary $A \beta$-amyloid in microscopic brain tissue sections, was $N$-methyl- $\left[{ }^{11} \mathrm{C}\right] 2-\left(4^{\prime}\right.$-methylaminophenyl)-6hydroxybenzothiazole (now termed $\left[{ }^{11} \mathrm{C}\right]$ Pittsburgh Compound-B or $\left.\left[{ }^{11} \mathrm{C}\right] \mathrm{PiB}\right)$ [1]. Shortly following the advent of $\left[{ }^{11} \mathrm{C}\right] \mathrm{PiB}$, several commercial entities began development of radio-fluorinated amyloid probes that would have potential translational clinical uses. At the time of this writing, there are a few probes approved for clinical use by Food and Drug Administration (FDA) and European Medicine Agency (EMA) and several others still in the pipeline.

As today, based on the available data, results obtained by PET with amyloid radiopharmaceuticals should not be considered as diagnostic for Alzheimer disease (AD), but rather indicative of the presence of cerebral amyloid deposition. $A \beta$ deposition may be found in fact in disorders other than $\mathrm{AD}$, such as dementia with Lewy bodies and amyloid angiopathy. Further researche will solve these issues, possibly increasing the spectrum of applications for amyloid PET imaging.

The Society of Nuclear Medicine and Molecular Imaging has recently released Guidelines for the clinical

K. Frey

University of Michigan, Ann Arbor, MI, USA

D. Perani $(\bowtie)$

Vita-Salute San Raffaele University and San Raffaele Hospital, Milan, Italy

e-mail: perani.daniela@hsr.it application of amyloid imaging, emphasizing a conservative approach [2]. The SNMMI Guidelines suggest appropriate use of clinical amyloid imaging for distinguishing dementias with a moderate-to-high burden of fibrillary $A \beta-$ amyloid vs. those without amyloid deposition. The specificities of PET findings with the use of the clinically approved tracers have each been confirmed by direct comparisons of PET imaging with subsequent neuropathologic diagnosis at autopsy. Patients with dementia due to Alzheimer disease and some patients with dementia with Lewy bodies are expected to have abnormal ("positive") amyloid scans, while patients with other forms of neurodegenerative disorders, such as the frontotemporal dementia spectrum (FTD), Creutzfeld-Jacob disease, and others, to have normal ("negative") amyloid scans.

Amyloid imaging might be of prognostic value in subjects with mild cognitive impairment (MCI), in whom there is a significant increased risk for progression to $\mathrm{AD}$ over 2 -3 years following a "positive" amyloid PET scan. On the other hand, amyloid imaging is appropriate neither in patients with typical $\mathrm{AD}$ and in asymptomatic elderly subjects, as the imaging results would not lead to changes in diagnostic classification or clinical management, in particular, "positive" amyloid scans may be observed in elderly individuals, that remain asymptomatic for a decade in spite of such finding. It remains possible that some of these individuals may never develop dementia-follow-up research studies are underway at present to evaluate this possibility. Conversely, when patients exhibit deficits and course that are typical of $\mathrm{AD}$, the vast majority will demonstrate "positive" amyloid scans, and the incremental contribution to diagnosis and management will be small.

Up to now, the types of patients and clinical circumstances in which amyloid PET imaging could be used are limited to patients with atypical dementia or in clinical 
trials, but only in conjunction with the other biomarkers, particularly CSF measures and FDG-PET. On the basis of recent FDG-PET imaging findings showing that neuronal injury can be early and independent of amyloid- $\beta$ deposition, a more complex picture [3] has progressively replaced the $\mathrm{A} \beta$ cascade hypothesis. The different molecular paths that PET in vivo biomarkers can reveal in the timeframe of $\mathrm{AD}$ progression reflect the events, leading to deposition of $\mathrm{A} \beta$ and phosphorylated tau, neuronal injury and neurodegeneration that can run in parallel instead than in a sequential manner. The amyloid and neuronal injury paths may diverge along the $\mathrm{AD}$ cascade, and bear separate relationships with $\mathrm{AD}$ symptoms and clinical phenotypes. All these evidences are crucial for the early diagnosis and for the development of new drugs aimed at slowing or preventing dementia. In this context, it is important to draw a clear distinction between the value of $A \beta$ imaging and the $A \beta$ hypothesis. The feasibility and validity of $A \beta$ imaging remains since, by definition, $A \beta$ deposition is a pathological hallmark of AD.

We are expecting further discussion regarding clinical indications for $\mathrm{A} \beta$ imaging and the debate about the causal role of $\mathrm{A} \beta$ pathology in the genesis of $\mathrm{AD}$ is one of the most important. The amyloid accumulation period can last for up to 15 years and during this period the positivity level gradually increases. It is thus common wisdom that a dichotomous classification (positive/negative exam) is inadequate for a thorough interpretation and reporting procedure. Therefore, a quantification of the exam providing information about the level of positivity is mandatory. The measurement has to be performed through adequate and innovative quantification tools, adapted to validated and standardized criteria.

We should also consider that different conformations of $\mathrm{A} \beta$ deposits in the brain may affect the uptake and retention pattern of the tracers and that $A \beta$ imaging modalities may not recognize all types of $A \beta$ pathologies with equal sensitivity. This may be an interesting area of future research, to further improve the understanding of the quantitative information provided by in vivo $A \beta$ imaging methods.

There are in addition several issues raised in the related literature out of the diagnostic value of $A \beta$ imaging, which require careful consideration and research. Among the most relevant, the nonspecific white matter retention and the role of $\mathrm{A} \beta$ pathology in $\mathrm{AD}$ disease generation. It is clear that the white matter uptake and the corresponding partial volume effects may lead to inaccuracies in the precise quantification of cortical tracer retention and thus assessment of cortical $A \beta$. While common to all $A \beta$ radiotracers, this is more noticeable in currently published studies using ${ }^{18} \mathrm{~F}$-labeled $\mathrm{A} \beta$ radiopharmaceuticals, that appear to generally show somewhat higher white matter retention as compared to $\left[{ }^{11} \mathrm{C}\right] \mathrm{PiB}$.
Further improvement in this context will regard the development of radiopharmaceuticals with less white matter retention and of image evaluation techniques, such as partial volume correction algorithms/volume of interest-based techniques for selective identification of gray matter uptake.

There is also a long-recognized discrepancy between cognitive impairment and $A \beta$ plaque burden in the brain. These mismatches between the presence of cerebral $\beta$-amyloidosis and symptoms of dementia paved the way to researches on the role of Amyloid-PET and FDG-PET imaging as conveying different molecular information on paths that can diverge along the timeframe of Alzheimer's disease progression. Amyloid-dependent and amyloid-independent mechanisms exist, and are not mutually exclusive, both contributing to Alzheimer's disease disorder. Designing interventional strategies that target the right molecular pathways at an appropriate stage of disease depends on accurate models of Alzheimer's disease biomarker evolution.

Although both FDA and EMA have approved PET imaging for the assessment of brain amyloid load, reimbursement is still at stake. At the present time healthcare insurance providers in the US do not offer reimbursement for amyloid imaging, while in Europe, where national health systems are in place in many countries, reimbursement is being debated. The argument is made that $\mathrm{AD}$ and other neurodegenerations cannot be effectively treated, and that improved survival and quality of life are not yet demonstrated to result from dementia management alternatives. Under the evidence that in turn amyloid imaging may not have a "therapeutic impact" its use would not be appropriate on the premises that the use of a diagnostic radiopharmaceutical, similarly to any other medicine, would be indicated under the assumption that it can produce an effect on patient outcome. This reasoning discounts entirely the value of diagnosis and prognosis afforded by amyloid imaging. Of even greater concern here, is the missed opportunity to discover potential management approaches and therapies that could affect the course of dementias. Clinical diagnostic accuracy in comparison to autopsy confirmation demonstrates accuracy in the range of $\sim 75 \%$, even in the context of expert clinicians in dedicated specialty clinics. Accuracy is expected to be even less when patients present early in the course of their cognitive decline, when specific signs or symptoms atypical of AD may be encountered. However, our ability to make needed progress in the discovery of effective therapies for dementias will rely heavily on accurate diagnostic classifications, together with the investigation of targeted new treatment approaches. A historical parallel to the current amyloid-imaging situation is the status of MRI for the diagnostic evaluation of suspected demyelinating disease in the 1980s. Use of MRI increased the sensitivity and accuracy of the diagnosis of multiple sclerosis, and 
contributed directly to the development of current effective immunomodulatory therapies. A similar breakthrough in the treatment of neurodegenerative dementias is overdue, and the now available contribution of molecular endophenotyping with amyloid-avid tracers will play a crucial role.

Conflict of interest The authors declare no conflict of interest.

\section{References}

1. Klunk WE, Engler H, Nordberg A, Wang Y, Blomqvist G, Holt DP, Bergström M, Savitcheva I, Huang G-F, Estrada S, Ausén B,
Debnath ML, Barletta J, Price JC, Sandell J, Lopresti BJ, Wall A, Koivisto P, Antoni GG, Mathis CA, Långström B (2004) Imaging brain amyloid in Alzheimer's disease with Pittsburgh compoundB. Ann Neurol 55:306-319

2. Johnson KA, Minoshima S, Bohnen NI, Donohoe KJ, Foster NL, Herscovitch P, Karlawish JH, Rowe CC, Carrilloi MC, Hartley DM, Hedrick S, Pappas V, Thies WH (2013) Appropriate use criteria for amyloid PET: a report of the Amyloid Imaging Task Force, the Society of Nuclear Medicine and Molecular Imaging, and the Alzheimer's Association. J Nucl Med 54:476-490

3. Jack CR Jr, Holtzman DM (2013) Biomarker modeling of Alzheimer's disease. Neuron 80(6):1347-1358 